\title{
Epigenetic changes in virus-associated human cancers
}

\author{
Hsin Pai LI ${ }^{1}$, Yu Wei LEU ${ }^{2}$,Yu Sun $\mathrm{CHANG}^{1^{*}}$ \\ ${ }^{1}$ Graduate Institute of Basic Medical Sciences, Chang Gung University, Kwei-shan, Taoyuan, Taiwan 333. \\ ${ }^{2}$ Department of Life Science and Institute of Molecular Biology, ChungCheng University, Ming-Hsiung, Chia-Yi, Taiwan 621.
}

\begin{abstract}
Epigenetics of human cancer becomes an area of emerging research direction due to a growing understanding of specific epigenetic pathways and rapid development of detection technologies. Aberrant promoter hypermethylation is a prevalent phenonmena in human cancers. Tumor suppressor genes are often hypermethylated due to the increased activity or deregulation of DNMTs. Increasing evidence also reveals that viral genes are one of the key players in regulating DNA methylation. In this review, we will focus on hypermethylation and tumor suppressor gene silencing and the signal pathways that are involved, particularly in cancers closely associated with the hepatitis B virus, simian virus 40 (SV40), and Epstein-Barr virus. In addition, we will discuss current technologies for genome-wide detection of epigenetically regulated targets, which allow for systematic DNA hypermethylation analysis. The study of epigenetic changes should provide a global view of gene profile in cancer, and epigenetic markers could be used for early detection, prognosis, and therapy of cancer.
\end{abstract}

Keywords: epigenetics, DNA methylation, cancer, HBV, SV40, EBV.

\section{INTRODUCTION}

Epigenetics is the study of DNA modifications, which can regulate gene expression without changeing DNA sequences. Accumulating evidence shows that epigenetic modifications have a crucial role in cancer development. These epigenetic modifications may induce the followings: hypomethylation leading to oncogene activation and chromosome instability; hypermethylation resulting in tumor suppressor gene silencing; chromatin remodeling and RNA-associated silencing. These modifications require the dynamic activity of DNA methyltransferases (DNMTs), histone modifying enzymes, and their corresponding interacting cellular factors. DNA methylation of $\mathrm{CpG}$ dinucleotides, which represent the target for the covalent modification of DNA [1], is one of the major mechanisms in the epigenetic regulation of genes. The methyl group protrudes from the cytosine nucleotide into the major

*Correspondence: Yu Sun CHANG

Tel: 886-3-211-8800 ext 5131

Fax: 886-3-211-8683

E-mail: ysc@mail.cgu.edu.tw groove of the DNA, giving two major effects, to displace transcription factors that normally bind to the DNA $[2,3]$ and to attract methyl-binding proteins, which are functionally associated with gene silencing and chromatin compaction [4], probably via interactions with complexes that modify the tails of histone proteins. On the other hand, histone proteins form octomers around DNA loops to form nucleosome, the individual packaging unit of genomic DNA. The histone tails that extrude from the nucleosomes can be modified by methylation [5], acetylation [6], phosphorylation [7], or ubiquitination [8] to creat potential combinations that have been referred as histone codes [9] in which gene regulatory information is concealed. Most recently, histone demethylase has been identified [10]. This enzyme is referred to as lysine specific demethylase 1 (LSD1) because it can demethylate a lysine on the tail of histone $\mathrm{H} 3$. The LSD1 is likely, through maintaining an unmethylated histone, to repress specific genes. .

Within the last few years, a number of reviews with an emphasis on various aspects of epigenetic modifications in cancer have been published [11-15]. In this review, we will focus on hypermethylation and tumor suppressor gene silencing in cancer development, particularly in virus-associated cancers. 


\section{DNA methyltransferases, tumor suppressor genes and} cancer development

The first indication that epigenetics plays a role in cancer was the discovery of altered gene methylation in colorectal cancers by Feinberg \& Vogelstein [16]. Methylation mediated by DNMTs, occurs at the C-5 position of cytosine residues present in $\mathrm{CpG}$ dinulceotides. $\mathrm{CpG}$ dinucleotides known as $\mathrm{CpG}$ islands are locally enriched in shorter stretches of DNA, ranging from 0.5 to several kilobases (kb) [17]. CpG islands are most commonly associated with promoter regions and present in approximately $60 \%$ of human genes [18]. DNA methylation of the $\mathrm{CpG}$ islands is mediated by at least three active DNA methyltransferases: DNMT1, DNMT3A, and DNMT3B. DNMT1 functions as a hemi-methylase responsible for maintenance of correct methylation patterns for DNA replication. DNMT3A and DNMT3B, on the other hand, are de novo methyltransferases important for establishing methylation patterns during embryogenesis.

DNMT1 is necessary and sufficient for Fos-induced transformation of mouse fibroblast cells [19]. DNMT1 knockdown experiments in human cancer cell lines have demonstrated an essential role for DNMT1 in maintaining aberrant repression of tumor suppressor loci [20]. Similarly, cell culture studies have demonstrated an important role for DNMT3A in cancer cell survival [21]. ICF syndrome, a rare disorder characterized by immunodeficiency, centromeric instability, and facial abnormalities, is caused by germ-line mutations in $d n m t 3 b[22$, 23]. Interestingly, mouse embryo fibroblasts deficient of DNMT3B are resistant to transformation by SV40 large $\mathrm{T}$ antigen and contain active Ras oncogenes [24], suggesting that epigenetic and genetic mechanisms likely act in concert during cellular transformation. It is also worth noting that DNMT1 and DNMT3B are overexpressed in certain human cancers, although only at moderate levels [25-27].

DNA methylation is tightly connected to cancer development. Oncogenesis is promoted by local hypermethylation of tumor-suppressor genes, whereas global genomic hypomethylation, on the other hand, affects oncogene expression and genomic stability [12]. Existing data suggest that the number of cancer-related genes affected by epigenetic inactivation equals to or exceeds the numbers that are inactivated by mutation $[12,28]$. Many genes modified by promoter hypermethylation have the classic tumor-suppressor function. Hypermethylation of tumor suppressor genes is generally associated with transcriptional repression to give rise to reduced expression or even gene silencing. Furthermore, hypermethylation of tumor suppressor genes can be an early event in cancer development [29-31] suggesting that both epigenetic modifications and genetic mutations can contribute to the disfunction of tumor suppressor genes, leading ultimately to tumor formation.

\section{VIRUS INFECTION, EPIGENETICS ALTER- ATIONS AND CARCINOGENESIS}

Virus infection especially DNA viruses and retroviruses, which may cause insertion of viral DNA sequence into the host genome, often triggers the host defense mechanism, particularly, DNA methylation machinery, to cause the methylation of foreign movable viral genome. DNA methylation of these viral DNAs is an effective method to silence viral gene expression. This process catalyzed by DNMTs involves the addition of methyl group in the cytosine residue primarily onto the viral DNA promoter $\mathrm{CpG}$ islands. Methylation labels the viral genome, and this in turn, recruits other protein factors such as methyl CpG binding protein (MeCP) 1 and 2, histone deacetylases (HDACs) [32] and transcription repressor (Sin3) [33] to form specific protein complex on methylated $\mathrm{CpG}$ island containing promoter. These regions are inaccessible to transcription factors, while transcription repressors on the other hand are recruited to the protein complex. Chromatin structure becomes compacted due to histone deacetylation. Consequently, gene transcription is inhibited or silenced. To maintain the genome stability, DNA methylation induced gene silencing on the integrated virus DNA is crucial for mammalian cells to subdue the intruder's DNA. Exogenous retroviral DNAs can be inactivated due to de novo DNA methylation and were able to integrate into human genome to become part of it [34]. Indeed, human genome contains DNA sequences resembling the retroviral long terminal repeats (LTRs) [35] and the transposable elements. Interestingly, viruses can find ways to counteract this cellbased modification and even take advantages of it. An informative review has discussed how could different viruses adapt different tactics to regulate viral gene expression through DNA methylation [36]. DNA methylation, in general, suppresses the viral gene expression, yet, methylation of distinct sets of gene promoters in EpsteinBarr virus (EBV) signifies different latency stages (I-III), which is essential for EBV to establish persistent infection and helps virus to evade the host immune system [37]. It is conceivable that once the viral genes are modified, it may as well affect the methylation status of the host genome.

Virus infection is one of the many factors having been linked to the development of various human cancers. Since virus genome may cause disruption of the host genome by insertion mutations and chromosomal rearrangements, cells infected by virus can be predisposed into cancer. For example, an insertion of viral promoter sequences adja- 
cent to host protooncogenes can cause neoplasia. Some DNA and RNA viruses may even carry their own viral oncogenes with transforming activities. Apart from introducing genetic changes, the presence of viral genome correlates to the aberrant methylation profile in human cancers. However, little is known about the molecular basis of virus-associated methylation. Here, three viruses, hepatitis B virus, Simian virus 40 and EBV, have been chosen to demonstrate their roles in altering host genome methylation profile. Such epigenetic modification of virus on cellular genes may contribute to viral pathogenesis and tumorigenesis. More importantly this deregulated methylation can be induced by the upregulation of the key methylation enzymes DNMTs, especially in cells expressing EBV oncoprotein latent membrane protein 1 (LMP1). Abnormal methylation can predispose cells into precancerous stage through inactivation of tumor suppressor genes and cell cycle regulated genes by hypermethylation, and reactivation of oncogenes by hypomethylation within the promoter region. We will also discuss the signaling pathways involved in DNMTs activation, and the role of epigenetic alteration in virus-induced carcinogenesis.

\section{Hepatitis B virus}

HBV is a member of hepadnaviruses, which contains a double-stranded circular DNA genome of $3.2 \mathrm{~kb}$. Integrated HBV genome is frequently detected in chronic virus infection and hepatocellular carcinoma (HCC) patients. Epidemiology data have shown that $\mathrm{HBV}$ is one of the etiologic agents in HCC development. However, the molecular mechanism(s) of HBV leading to HCC remains unclear. A well-accepted "indirect" model suggested that $\mathrm{HBV}$-induced cell injury, the result of immune responses to HBV infection, causes liver regeneration [38]. Intensive cell proliferation during liver cell regeneration accumulates mutations, increasing chances of errors during DNA replication. Cells acquired appropriate genetic mutations will undergo clonal expansion and finally develop into HCC.

Several studies have shown that a tumor suppressor gene, $p 16^{I N K 4}$ is frequently inactivated $(\sim 60 \%)$ in HBVrelated $\mathrm{HCC}$ tumor specimens due to extensive $\mathrm{CpG}$ methylation within the promoter region [39-41]. The gene product $\mathrm{p} 16^{\mathrm{INK} 4}$ acts as an inhibitor of cyclin-dependent kinase (CDK) 4 and 6 and inhibits the kinase activity of CDK $4 / 6$ on retinoblastoma $(R B)$ protein to cause $G_{1}$-arrest. Inactivation of $p 16^{I N K 4}$ in neoplastic cells promotes uncontrolled cell proliferate. Methylated $p 16^{I N K 4}$ is even present in chronic hepatitis and cirrhosis but with relatively lower frequency $(\sim 15 \%)$ [41], indicating that inactivation of $p 16$ ${ }^{I N K 4}$ may occur in early precancerous stage. Therefore, hepatitis virus infection may be one of the risk factors for inactivation of the $p 16^{I N K 4}$ via hypermethylation.

In one report, the promoter region of glutathione Stransferase (GSTP1), encoding an enzyme that protects cells from oxidation damage and electrophilic carcinogens, is commonly hypermethylated $(65 \%)$ in hepatitis B virus (HBV)-associated HCC tissues [42]. In those cases, GSTP1 protein is absent. Thus, HBV-related HCC is highly linked to the hypermethylation of cellular genes $p 16^{I N K 4}$ and GSTP1. However, it is still not known which HBV viral gene is responsible for $\mathrm{CpG}$ hypermethylation. Whether HBV genome has a direct effect on the $\mathrm{CpG}$ methylation of these gene promoter remains ambiguous. Therefore, one cannot rule out the possibility that HBV infection and CpG methylation in $p 16^{I N K 4}$ and GSTP1 are independent events during hepatocarcinogenesis.

To examine global epigenetic and genetic alterations associated with the HBV integration in HCC, restriction landmark genomic scanning (RLGS), which is a two-dimensional gel electrophroesis system, was used to compare genomic DNA profiles of HCCs with that of the cell lines with or without HBV integration [43]. According to this study, one NotI landmark spot appeared in HBV-integrated cell lines or in primary HCCs, but not in HCCs or tumorcell lines without HBV integration. Cloning of this spot revealed that it comprised of a NotI cluster sequence with $\mathrm{CpG}$ dinucleotides. This sequence, located to the peri-centromeric region, was hypomethylated in association with HBV integration. The results demonstrated that epigenetic changes at specific position in the genome accompanied with HBV integration may be related to hepatocarcinogenesis. To evaluate whether epigenetic change, particularly aberrant DNA methylation, is a cause or consequence of tumorigenesis, mice with reduced DNMT expression were established to examine the effect of genome-wide DNA hypomethylation [44, 45]. In these studies, mutant mice developed early onset of lymphoma and frequently show chromosomal abnormality such as chromosome trisomy, indicating that DNA hypomethylation indeed plays an important role in tumorigenesis, probably via promoting chromosomal instability. Hence, HBV-induced genome hypomethylation may be similar to that of the DNMT mutant mice. In both cases DNA hypomethylation may increase the risk of cancer by promoting genomic instability.

\section{Simian virus 40}

SV40 is a member of polyomavirus, with a small doublestranded, circular DNA genome of $5 \mathrm{~kb}$. SV40 is a tumor virus of primate origin. It has spread to human via contaminated poliovirus vaccines [46]. SV40 large T antigen is a viral protein required for viral DNA replication, and a potent transforming protein. It binds and inactivates 
tumor suppressor gene products, p53 and RB proteins. SV40 infection has been linked to a number of human cancers, including mesotheliomas, bone and brain tumors and lymphomas [47-50], although the mechanism involved is, as yet, controversial. In malignant mesothelioma (MM), a lethal neoplastic disease associated with asbestos exposure, SV40 DNA can be detected in 48\% of MM patients [48]. However, there is no apparent link between SV40 and asbestos exposure suggesting that both viral and environmental factors contribute to the development of MM.

Aberrant methylation of the tumor suppressor gene Rasassociation domain family $1 \mathrm{~A}(R A S S F 1 A)$ is detected in SV40-associated MM. By using methylation specific PCR, methylation percentage of four genes, hyperplastic polyps (HPP1), RASSF 1A, cyclin D2, and Ras-related associated with diabetes $(R R A D)$, was significantly higher in SV40-positive MMs than that in SV40-negative MMs [51]. To mimic the development of $\mathrm{MM}$, an in vitro cell culture system, SV40-infected normal human mesothelial cells (HM), was established [51]. Interestingly, SV40-infected HM showed progressive aberrant methylation in seven genes [RASSF1A, HPP1, Decoy receptor 1 (DcRl), target of methylation-induced silencing (TMS1), cellular retinolbinding protein $(C R B P 1)$, hypermethylated in cancer $(H I C-1)$, and $R R A D]$ especially after fifty serial cell passages, suggesting the accumulation of epigenetic errors overtime or during DNA replication. These results demonstrate a close link between SV40 and methylation of multiple genes in MM, indicating that SV40 plays a role in MM pathogenesis .

In addition to inactivate tumor suppressor gene, SV40 $\mathrm{T}$ antigen has been shown to act in concert with activated Ras and teleomerase to form colonies in soft agar assay by using normal human bronchial cells (NHBC) [24]. The expression of one of the DNMT 3B isoforms was increased in these transformed cells. The introduction of DNMT3B antisense RNA suppressed T antigen-induced transformation. Similarly, mouse embryo fibroblasts expressing $\mathrm{T}$ antigen and Ras formed soft agar colonies and tumors, yet fibroblasts from $D n m t 3 b^{-/-}$mice did not grow on soft agar. Furthermore, the transcription of two tumor suppressor genes, fragile histidine triad (FHIT) and tumor suppressor in lung cancer (TSLC1) were reduced in transformed NHBE cells, while DNMT3B antisense RNA reactivated the expression of FHIT and TSLC1. These results indicate that $\mathrm{T}$ antigen and Ras work together to activate DNMT3B, a de novo DNA methyltransferase. It is likely that the $\mathrm{CpG}$ islands on tumor suppressor genes' promoter regions become one of the targets of DNMT3B. Obviously, inactivation of tumor suppressor genes due to hypermethylation is one of the major reasons leading to tumor progression.

\section{Epstein-Barr virus}

EBV is a ubiquitous human herpesvirus, with a huge double-stranded DNA genome of $\sim 172 \mathrm{~kb}$. EBV is closely associated with several human malignancies, including nasopharyngeal carcinoma (NPC), Burkitt's lymphoma, $\mathrm{T}$ cell lymphoma and gastric carcinoma [52]. In NPC, EBV infection is predominantly latent, and viral gene expression is under strict control. During different latency phase, different viral promoter sets will be activated via regulation of methylation. One of the viral genes, the latent membrane protein 1 (LMP1), is expressed in $\sim 70 \%$ of NPC. LMP1 is an oncoprotein with transforming capability $[53,54]$. It has been shown that human epithelial cells expressing $L M P 1$ had higher invasive ability, in correlation with reduced E-cadherin expression $[55,56]$. Our previous data demonstrated that reduction of E-cadherin is the result of LMP1-induced hypermethylation of Ecadherin gene promoter through activation of DNMT1, $3 \mathrm{~A}$ and $3 \mathrm{~B}$ [57]. Addition of DNMT inhibitor, 5'-Aza-2'$\mathrm{dC}$, in LMP1 expressing epithelial cells, restored the expression of the E-cadherin, suggesting that LMP1 regulates the expression of specific cellular gene via epigenetic modification. Recently, we have defined the region on LMP1, which is responsible for the DNMT activation and the signaling pathway involved (Tsai et al in preparation). By using different LMP1 deletion mutants, we were able to locate the C-terminal activation region 2 (CTAR2) of LMP1 as the domain required for DNMT activation. It has been reported that CTAR2 can activate AP-1/JNK signaling pathway [58]. We thus use JNK inhibitor (SP60012) to show that DNMT is indeed the downstream target of JNK. In addition, mutation of the AP-1 site on DNMT1 promoter, and c-Jun dominant-negative mutant blocked the LMP1-induced DNMT activation. Chromatin immunoprecipitation (ChIP) assay further demonstrated that DNMT1, 3A, 3B, MeCP2 and HDAC1 formed protein complex on the E-cadherin promoter in the LMP1 positive cells but not in the control cells. Taken together, a single viral oncoprotein in EBV has been identified, which is capable of activating DNMTs via AP-1/JNK signaling pathway. DNMTs are key enzymes to inactivate transcription by DNA hypermethylation and to ensure proper gene repression. In order to maintain the harmonious internal environment, the protein level or the enzymatic activity of DNMTs should be tightly controlled. Nevertheless, disruption of this delicate balance will result in chaos. The fact that LMP1 activates DNMTs highlights the importance of virus as an initiator or a cofactor to induce epigenetic alterations. In fact, LMP1 is the first viral gene that was identified with the ability to activate DNMTs via a 
Epigenetic changes in virus-associated human cancers

defined signaling pathway. Aberrant DNA hypermethylation induced by viral gene is one of the methods that virus contributes to tumor development. Besides E-cadherin $(E-$ $\mathrm{cad}$ ) promoter, other tumor suppressor gene promoters such as $R A S S F 1 A$, retinoic acid receptor $(R A R) \beta 2, p 16^{I N K 4}$ and p14 are also hypermethylated in NPC [59-62], supporting the previous evidence that frequent hypermethylation on cellular promoters may be a common phenomenon in NPC.

Several clinical reports stated that EBV-associated gastric carcinoma (GC) exhibited global and non-random DNA methylation, including $p 16^{I N K 4}$ and E-cad [63-65]. Intriguingly, EBV-associated $\mathrm{GC}$ does express latency genes (EBNA1, EBER1, BARF0 and LMP2A), but does not express $L M P 1$ and EBNA2 [66], suggesting other viral gene(s) or alternative mechanism may be involved in the EBV-associated GC epigenetic alteration.

It appears that different viruses induce both genetic and epigenetics changes in the infected cells. Viruses utilize similar strategies to alter the epigenetic information of their hosts, and these strategies have been summarized in Fig. 1. Viral proteins are involved either directly or indirectly in activation of DNMTs, especially that of the DNMT3b, a gene required for de novo DNA methylation. Thus aberrant methylation or epigenetic silencing of the key regulators of cell growth, signaling pathways and tumor suppressor genes are the main causes for tumor formation. However, whether this methylation event has preference on certain genes remains unknown. In addition to SV40 and EBV, infection of HIV also causes upregulation of the DNMT1 protein expression $[67,68]$. This activation in turn elevated the global genomic methylation and on the other hand suppressed other genes expression such as interferon- $\gamma(I F N-\gamma)$, a cytokine important for immune response during virus infection [67], and pl $6^{I N K 4}$, a common altered tumor suppressor gene found in cancers. This aberrant methylation may correlate to AIDS and AIDSassociated malignancies; however, it is not clear which HIV viral gene is involved in such process. Nevertheless, increasing evidence has revealed that viral genes are one of the key players in regulation of DNMTs. Identification of the pathways involved in regulating DNMT by viral gene may gain insights into the virus-mediated pathogenesis and tumorigenesis.

\section{MOLECULAR TECHNOLOGY FOR CANCER EPIGENOMICS}

Epigenetic research offers a major opportunity for understanding a novel mechanism of gene regulation and its roles in cancer development. It is important to systematically identify potential methylation-specific targets, which

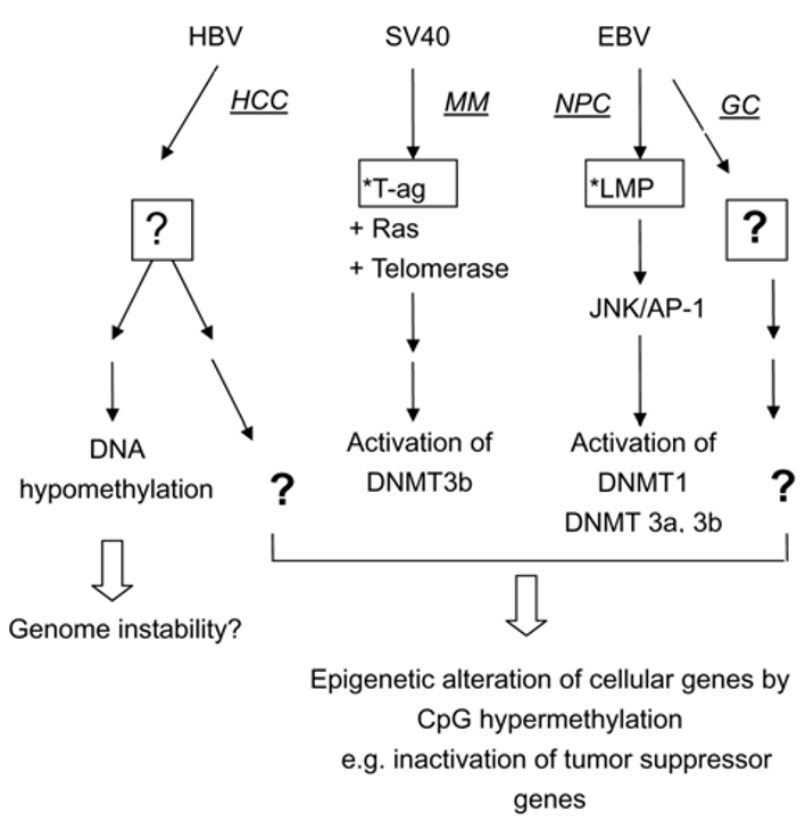

Fig. 1 Various strategies for viruses to alter host epigenetics via DNA methylation. Viruses silence cellular genes via DNA hypermethylation within the gene promoter region by direct activation of DNMTs or by some unknown mechanism (?). EBV oncoprotein LMP1 is a well-studied viral protein that can activate DNMT1, DNMT3a and 3b (Tsai and Chang, unpublished data), thereby altering the epigenetic state of cellular genes, especially tumor suppressor genes e.g. E-cadherin, $p 16$ and RASSF1A. Viral genes are enclosed in a rectangle. The asterisk represents the identified viral genes that are involved in regulating the activity of DNMTs. Virus associated human malignancies are shown in italics and underlined. HCC, hepatocarcinoma; MM, malignant mesotheliomas; NPC, nasopharyngeal carcinoma; $\mathrm{GC}$, gastric cancer.

could be served as biomarkers for cancer prevention, prognosis and treatment. Thus, in the following section, we will discuss current technologies used for identification of methylation-regulated genes. It has been established that DNA methylation can be mostly preserved in the clinical samples. In contrast, the detection of the chromatin modifications is less feasible in patients' samples. Cancer epigenomic measurement has been focused on the detection of the tumoral DNA methylation modifications. Therefore, the majority of the developed techniques are aiming for the characterization of single gene or genomewide DNA methylation.

\section{PCR-based single gene DNA methylation detection}

A number of PCR-based detection methods has been widely used to characterize single gene methylation modification. These include: (1) bisulfite DNA conversion $[69,70]$ followed by PCR, in which the methylated DNA 
is protected against the bisulfite treatment, whereas, the cytosine in the unmethylated DNA will be modified into uracil after bisulfite conversion. After PCR amplification uracil will further turn to thymidine; this conversion process creates a physical difference in unmethylated DNA sequence. However, DNA sequencing must be performed to confirm the sequence conversion. Essentially, most detection methods developed later on are based on this $\mathrm{C}$ to T conversion. (2) Modified bisulfite conversion in combination of restriction enzyme analysis called the Combined Bisulfite Restriction Analysis (COBRA) [71] is used to distinguish methylated versus unmethylated DNA by the loss of specific enzyme site e.g. BstI, in the unmethylated DNA after bisulfite conversion. (3) Methylation specific PCR (MSP) [72] uses specific methylated and unmethylated primers, whose sequences contain possible methylation sites to PCR amplify the bisulfite converted genomic DNA. Alternatively, a similar set of primers is designed to perform a more sensitive, high-throughput quantitative methylation assay that is fluorescence-based real-time PCR (TaqMan) technology "MethyLight" [73-75]. (4) Recently, two modified bisulfite-treated DNA methylation-mapping techniques have been developed; they are "pyrosequencing" $[76,77]$ and "RNase T1 cleavage/matrix-assisted laser desorption ionization time-of-flight (MALDI-TOF)" [78]. Pyrosequencing is a real-time sequencing technology, which detects the release of pyrophosphate (PPi) during nucleotide incorporation, the quantitative conversion of pyrophosphate to ATP by sulfurylase, and the subsequent production of visible light by luciferase [79]. This method can measure as many as 10 successive CpGs in a single sequencing reaction spanning up to 75 nucleotides. The MALDI-TOF approach requires the conversion of the bisulfite-treated DNA into RNA by tagging the PCR product with $\mathrm{T} 7$ promoter. The in vitro transcribed RNA is then subjected to RNaseT1 cleavage to produce a specific pattern of RNA fragments. These RNAs are analyzed using MALDDI-TOF technology to distinguish methylated and unmethylated DNA. These modified techniques provide a rapid analysis of $\mathrm{CpG}$ methylation patterns and may develop as a high-throughput screening platform.

\section{Genome-wide epigenomic detections}

Although single locus methylation is sufficient to disturb gene expression, tumorigenesis is often found to be associated with changes in global DNA methylation. Currently, the array based or non-array based technologies have been developed to detect this genome-wide methylation changes. Some of the non-array based methods, like HPLC, detect the overall content of the genome-wide DNA methylation without revealing the exact identifications of the genes. These methods provide a quick over- view of the genome's methylation status.

The other non-array based method like RLGS [80-82] uses the methylation-sensitive enzyme like Not $\mathrm{I}$ to create a genome-wide methylation differences between the tumor and non-tumor parts. These DNAs were then labeled with isotope, and profiled onto two reproducible two-dimensional gels. After comparison, the gained or lost spots can be isolated from the gels, recovered, cloned and sequenced. Progressive identifications of the frequent methylated and/ or de-methylated loci make the data processing easier and more reliable. The potential of cloning and arraying all of these NotI frequent methylated fragments makes the applications of the RLGS even broader. The methylationsensitive representational difference analysis (MS-RDA) [83] represents another type of methods that does not use microarray system. Still, these methods use the methylation sensitive enzyme to discriminate between the tumor and non-tumor samples, followed by the ligation and PCR process similar to subtraction PCR approach. The amplicons survived the subtraction are cloned and sequenced. Other similar methods have been adapted to scan the cancer methylation status without using the array system.

The array-based technology, or the CpG microarray system, stands out in interrogating the cancer epigenomics. The CpG islands are the hotspots for epigenetic regulation. At least three kinds of targets can be detected on this platform: (1) Differential methylation hybridization (DMH) [84-86] detects the cancer methylation status, (2) ECIST $[87,88]$ measures the gene transcripts, and the (3) ChIPon-chip [89] interrogates the chromatin conformational changes (Fig. 2). Since these three arrays are performed on the same platform, the results from different assay can be compared directly, and more reliable data will be obtained. Therefore, one set of data can be used to confirm with of the others. Since ChIP-on-chip microarray can be used to identify transcription factors binding on the target loci, it is possible to monitor the epigenetic modifications within certain signaling pathways [90] and to dissect the cancer epigenomic pathway systematically.

Technologies are rapidly evolving to probe the cancer epigenetic modifications, and the accuracy and sensitivity of these methods are improving. Moreover, the majority of these measurements are now equipped with high throughput scanning ability to accelerate the screening process. Screening process can be modified by focusing on a smaller panel of CpG array with biological-related specific genes. Hence it may speed up the discovery of epigenetically regulated biomarkers in a given cancer.

\section{PERSPECTIVES}

Methylation of DNA has been shown to be the most consistent molecular change in many neoplasms. DNA 


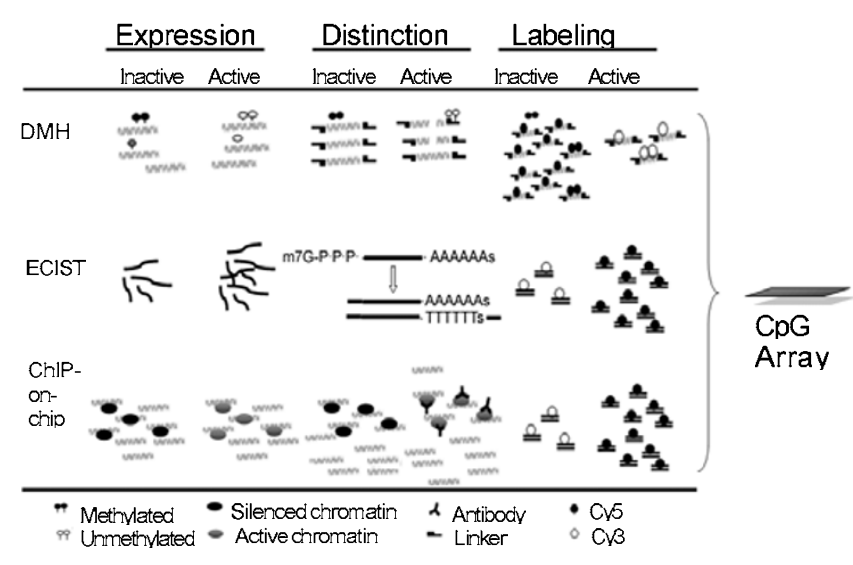

Fig. 2 DMH, ECIST and ChIP-on-chip analysis of the cancer epigenome using $\mathrm{CpG}$ island microarray. Three microarray systems are developed to target the same $\mathrm{CpG}$ island array and to detect changes in DNA methylation or chromatin conformation (ChIP-onchip) status during cancer progression. Experimental data obtained from one screening system can be validated by the other two systems. Differential methylation hybridization (DMH) is used to detect global DNA methylation changes. Tumor genomic DNA is digested into small fragments and ligated with adaptors. Methylation sensitive restriction enzymes (e.g. Bst $\mathrm{UI}$ and $H p a \mathrm{II}$ ) are used to discriminate the methylated and unmethylated DNA fragments. Differences in methylation status are amplified by PCR using the adaptor sequence as primers. The amplified signals from tumor and non-tumor are labeled with $\mathrm{Cy} 5$ and $\mathrm{Cy} 3$ dyes, respectively, and co-hybridized onto the CpG microarray. The second system is the "expressed CpG island sequence tag" (ECIST). Profile of gene expression can be obtained by hybridizing CpG microarray with cDNA, which is enriched at the 5'-end of the expressed genes by RNA ligase-mediated cDNA synthesis, RLCS [51]. As a result, a methylated locus usually signifies a silenced gene. The third system is ChIP (Chromatin immunoprecipitation)-on-chip microarray [89], which is developed to dissect the tumoral chromatin conformation and/or upstream transcriptional activities directly. Antibodies against specific modified chromatin components such as Acetyl-Histone 3 (correlated with active transcriptional activities) can be used to immunoprecipitate the chromatin component in an active/silent status. After purification, the stripped DNA are labeled and hybridized with the CpG array.

methylation results in hypermethylation of specific target genes, which can be detected at various stages of cancer development. Hopefully, in the near future, we can identify signature hypermethylated genes that can be used as biomarkers for specific type of cancer.

Current techniques are focused on identifying the presence of disease, and to a much lesser extent, on early detection of malignancies. An epigenetic alteration might function as a marker prior to the development of cancer [91-93]. Thus, epigenetic markers may be crucial for identifying the risk of cancer development. The systematic approaches described above may allow one to identify those novel targets regulated through epigenetic modifications.

An epigenetic change, unlike a genetic change, can be easily reversed by the use of therapeutic interventions. Hypermethylated genes found in cancer seem to be particularly sensitive to reactivation by DNA methylation inhibitors. Therefore, therapeutic targeting DNA methylation may restore multiple gene functions at the same time. To date, many chemical agents have been discovered to selectively inhibit either DNMTs or HDACs. ADNA methylation inhibitor, 5-aza-2'-deoxycytidine, has been shown to restore a normal pattern of imprinting in tumor cells, without disrupting imprinting on normally marked alleles [94]. Two azanucleotides (5'-azacytidine, 5 -azaCdR) have entered clinical trials for treating patients with myeloid disease [95].

As described in the earlier section, viral proteins may regulate epigenetic modifications in virus-associated cancers. This provides a specific therapeutic strategy that targets both viral proteins and DNA methylation mediated by these proteins. Using both viral and methylated cellular genes as therapeutic markers may provide another option for interventions to prevent further progression in virusassociated cancers.

The significance of epigenetics in cancer development is clearly evident. The challenge in the research and identification of methylation-related target genes lie in the fact that methylation information is not retained during an amplification step (amplification by PCR or by cultivation as plasmid in E. coli). Although current systematic approaches have been proven to be successful in identification of methylation-regulated markers, the process is both time-consuming and labor-intensive. Technical improvements in the study of methylation should stimulate much future research on epigenetics.

\section{REFERENCES}

1 Sinsheimer RL. The action of pancreatic deoxyribonuclease. II. Isomeric dinucleotides. J Biol Chem 1955;215:579-83.

2 Hark AT, Schoenherr CJ, Katz DJ, et al. CTCF mediates methylation-sensitive enhancer-blocking activity at the H19/Igf2 locus. Nature 2000;405:486-9.

3 Kim J, Kollhoff A, Bergmann A, Stubbs L. Methylation-sensitive binding of transcription factor YY1 to an insulator sequence within the paternally expressed imprinted gene, Peg3. Hum Mol Genet 2003;12:233-45.

4 Bird AP, Wolffe AP. Methylation-induced repression-belts, braces, and chromatin. Cell 1999;99:451-4.

5 Sims RJ 3rd, Nishioka K, Reinberg D. Histone lysine methylation: a signature for chromatin function. Trends Genet 2003;19:62939.

6 Roth SY, Denu JM, Allis CD. Histone acetyltransferases. Annu Rev Biochem 2001;70:81-120.

7 Thomson S, Clayton AL, Mahadevan LC. Independent dynamic regulation of histone phosphorylation and acetylation during 
immediate-early gene induction. Mol Cell 2001;8:1231-41.

8 Zhang Y. Transcriptional regulation by histone ubiquitination and deubiquitination. Genes Dev 2003;17:2733-40.

9 Jenuwein T, Allis CD. Translating the histone code. Science 2001;293:1074-80.

10 Shi Y, Lan F, Matson C, et al. Histone demethylation mediated by the nuclear amine oxidase homolog LSD1. Cell 2004;119:94153.

11 Robertson KD. DNA methylation, methyltransferases, and cancer. Oncogene 2001;20:3139-55.

12 Jones PA, Baylin SB. The fundamental role of epigenetic events in cancer. Nat Rev Genet 2002;3:415-28.

13 Feinberg AP, Tycko B. The history of cancer epigenetics. Nat Rev Cancer 2004;4:143-53.

14 Egger G, Liang G, Aparicio A, Jones PA. Epigenetics in human disease and prospects for epigenetic therapy. Nature 2004;429: 457-63.

15 Lund AH, van Lohuizen M. Epigenetics and cancer. Genes Dev 2004;18:2315-35.

16 Feinberg AP, Vogelstein B. Hypomethylation distinguishes genes of some human cancers from their normal counterparts. Nature 1983;301:89-92.

17 Bird A. DNA methylation patterns and epigenetic memory. Genes Dev 2002;16:6-21.

18 Antequera F, Bird A. Number of CpG islands and genes in human and mouse. Proc Natl Acad Sci U S A 1993;90:11995-9.

19 Bakin AV, Curran T. Role of DNA 5-methylcytosine transferase in cell transformation by fos. Science 1999;283:387-90.

20 Robert MF, Morin S, Beaulieu N, et al. DNMT1 is required to maintain CpG methylation and aberrant gene silencing in human cancer cells. Nat Genet 2003;33:61-5.

21 Beaulieu N, Morin S, Chute IC, et al. An essential role for DNA methyltransferase DNMT3B in cancer cell survival. J Biol Chem 2002;277:28176-81.

22 Hansen RS, Wijmenga C, Luo P, et al. The DNMT3B DNA methyltransferase gene is mutated in the ICF immunodeficiency syndrome. Proc Natl Acad Sci U S A 1999;96:14412-7.

$23 \mathrm{Xu} \mathrm{GL}$, Bestor TH, Bourc'his D, et al. Chromosome instability and immunodeficiency syndrome caused by mutations in a DNA methyltransferase gene. Nature 1999;402:187-91.

24 Soejima K, Fang W, Rollins BJ. DNA methyltransferase 3b contributes to oncogenic transformation induced by SV40T antigen and activated Ras. Oncogene 2003;22:4723-33.

25 De Marzo AM, Marchi VL, Yang ES, et al. Abnormal regulation of DNA methyltransferase expression during colorectal carcinogenesis. Cancer Res 1999;59:3855-60.

26 Girault I, Tozlu S, Lidereau R, Bieche I. Expression analysis of DNA methyltransferases $1,3 \mathrm{~A}$, and 3B in sporadic breast carcinomas. Clin Cancer Res 2003;9:4415-22.

27 Nagai M, Nakamura A, Makino R, Mitamura K. Expression of DNA (5-cytosin)-methyltransferases (DNMTs) in hepatocellular carcinomas. Hepatol Res 2003;26:186-191.

28 Herman JG, Baylin SB. Gene silencing in cancer in association with promoter hypermethylation. N Engl J Med 2003;349:204254.

29 Waki T, Tamura G, Sato M, et al. Promoter methylation status of DAP-kinase and RUNX3 genes in neoplastic and non-neoplastic gastric epithelia. Cancer Sci 2003;94:360-4.

30 Lee JH, Park SJ, Abraham SC, et al. Frequent CpG island me- thylation in precursor lesions and early gastric adenocarcinomas. Oncogene 2004;23:4646-54.

31 Suter CM, Martin DI, Ward RL. Germline epimutation of MLH1 in individuals with multiple cancers. Nat Genet 2004;36:497501.

32 Jones PL, Veenstra GJ, Wade PA, et al. Methylated DNA and $\mathrm{MeCP} 2$ recruit histone deacetylase to repress transcription. Nat Genet 1998;19:187-91.

33 Heinzel T, Lavinsky RM, Mullen TM, et al. A complex containing $\mathrm{N}-\mathrm{CoR}, \mathrm{mSin} 3$ and histone deacetylase mediates transcriptional repression. Nature 1997;387:43-8.

34 Yoder JA, Walsh CP, Bestor TH. Cytosine methylation and the ecology of intragenomic parasites. Trends Genet 1997;13:33540.

35 Lavie L, Kitova M, Maldener E, Meese E, Mayer J. CpG methylation directly regulates transcriptional activity of the human endogenous retrovirus family HERV-K(HML-2). J Virol 2005; 79:876-83.

36 Verma M. Viral genes and methylation. Ann N Y Acad Sci 2003; 983:170-80.

37 Tao Q, Robertson KD. Stealth technology: how Epstein-Barr virus utilizes DNA methylation to cloak itself from immune detection. Clin Immunol 2003;109:53-63.

38 Chisari FV, Ferrari C. Hepatitis B virus immunopathogenesis. Annu Rev Immunol 1995;13:29-60.

39 Shim YH, Yoon GS, Choi HJ, Chung YH, Yu E. p16 Hypermethylation in the early stage of hepatitis B virus-associated hepatocarcinogenesis. Cancer Lett 2003;190:213-9.

40 Narimatsu T, Tamori A, Koh N, et al. p16 promoter hypermethylation in human hepatocellular carcinoma with or without hepatitis virus infection. Intervirology 2004;47:26-31.

41 Li X, Hui AM, Sun L, et al. p16INK4A hypermethylation is associated with hepatitis virus infection, age, and gender in hepatocellular carcinoma. Clin Cancer Res 2004;10:7484-9.

42 Zhong S, Tang MW, Yeo W, et al. Silencing of GSTP1 gene by CpG island DNA hypermethylation in HBV-associated hepatocellular carcinomas. Clin Cancer Res 2002;8:1087-92.

43 Nagai H, Baba M, Konishi N, et al. Isolation of NotI clusters hypomethylated in HBV-integrated hepatocellular carcinomas by two-dimensional electrophoresis. DNA Res 1999;6: 219-25.

44 Gaudet F, Hodgson JG, Eden A, et al. Induction of tumors in mice by genomic hypomethylation. Science $2003 ; 300: 489-92$.

45 Eden A, Gaudet F, Waghmare A, Jaenisch R. Chromosomal instability and tumors promoted by DNA hypomethylation. Science 2003;300:455.

46 Butel JS. Simian virus 40, poliovirus vaccines, and human cancer: research progress versus media and public interests. Bull World Health Organ 2000;78:195-8.

47 Shivapurkar N, Wiethege T, Wistuba II, et al. Presence of simian virus 40 sequences in malignant pleural, peritoneal and noninvasive mesotheliomas. Int J Cancer 2000;85:743-5.

48 Shivapurkar N, Wiethege T, Wistuba II, et al. Presence of simian virus 40 sequences in malignant mesotheliomas and mesothelial cell proliferations. J Cell Biochem 1999;76:181-8.

49 Shivapurkar N, Harada K, Reddy J, et al. Presence of simian virus 40 DNA sequences in human lymphomas. Lancet 2002; 359:851-2.

50 Gazdar AF, Butel JS, Carbone M. SV40 and human tumours: 
myth, association or causality? Nat Rev Cancer 2002;2:957-64.

51 Suzuki M, Toyooka S, Shivapurkar N, et al. Aberrant methylation profile of human malignant mesotheliomas and its relationship to SV40 infection. Oncogene 2005;24:1302-8.

52 Shibata D, Weiss LM. Epstein-Barr virus-associated gastric adenocarcinoma. Am J Pathol 1992;140:769-74.

53 Wang D, Liebowitz D, Kieff E. An EBV membrane protein expressed in immortalized lymphocytes transforms established rodent cells. Cell 1985;43:831-40.

54 Fahraeus R, Rymo L, Rhim JS, Klein G. Morphological transformation of human keratinocytes expressing the LMP gene of Epstein-Barr virus. Nature 1990;345:447-9.

55 Fahraeus R, Chen W, Trivedi P, Klein G, Obrink B. Decreased expression of E-cadherin and increased invasive capacity in EBVLMP-transfected human epithelial and murine adenocarcinoma cells. Int J Cancer 1992;52:834-8.

56 Kim KR, Yoshizaki T, Miyamori H, et al. Transformation of Madin-Darby canine kidney (MDCK) epithelial cells by EpsteinBarr virus latent membrane protein 1 (LMP1) induces expression of Ets1 and invasive growth. Oncogene 2000;19:1764-71.

57 Tsai CN, Tsai CL, Tse KP, Chang HY, Chang YS. The EpsteinBarr virus oncogene product, latent membrane protein 1, induces the downregulation of E-cadherin gene expression via activation of DNA methyltransferases. Proc Natl Acad Sci U S A 2002;99: 10084-9.

58 Kieser A, Kaiser C, Hammerschmidt W. LMP1 signal transduction differs substantially from TNF receptor 1 signaling in the molecular functions of TRADD and TRAF2. Embo J 1999;18: 2511-21.

59 Lo KW, Kwong J, Hui AB, et al. High frequency of promoter hypermethylation of RASSF1A in nasopharyngeal carcinoma. Cancer Res 2001;61:3877-81.

60 Lo KW, Huang DP. Genetic and epigenetic changes in nasopharyngeal carcinoma. Semin Cancer Biol 2002;12:451-62.

61 Kwong J, Lo KW, To KF, et al. Promoter hypermethylation of multiple genes in nasopharyngeal carcinoma. Clin Cancer Res 2002;8:131-7.

62 Tong JH, Tsang RK, Lo KW, et al. Quantitative Epstein-Barr virus DNA analysis and detection of gene promoter hypermethylation in nasopharyngeal (NP) brushing samples from patients with NP carcinoma. Clin Cancer Res 2002;8:2612-9.

63 Kang GH, Lee S, Kim WH, et al. Epstein-barr virus-positive gastric carcinoma demonstrates frequent aberrant methylation of multiple genes and constitutes $\mathrm{CpG}$ island methylator phenotype-positive gastric carcinoma. Am J Pathol 2002;160:787-94.

64 Chong JM, Sakuma K, Sudo M, et al. Global and non-random CpG-island methylation in gastric carcinoma associated with Epstein-Barr virus. Cancer Sci 2003;94:76-80.

65 Sakuma K, Chong JM, Sudo M, et al. High-density methylation of p14ARF and p16 ${ }^{\mathrm{INK} 4 \mathrm{~A}}$ in Epstein-Barr virus-associated gastric carcinoma. Int J Cancer 2004;112:273-8.

66 Imai S, Koizumi S, Sugiura M, et al. Gastric carcinoma: monoclonal epithelial malignant cells expressing Epstein-Barr virus latent infection protein. Proc Natl Acad Sci U S A 1994;91:9131-5.

67 Mikovits JA, Young HA, Vertino P, et al. Infection with human immunodeficiency virus type 1 upregulates DNA methyltransferase, resulting in de novo methylation of the gamma interferon (IFN- $\gamma$ ) promoter and subsequent downregulation of IFN$\gamma$ production. Mol Cell Biol 1998;18:5166-77.
68 Fang JY, Mikovits JA, Bagni R, Petrow-Sadowski CL, Ruscetti FW. Infection of lymphoid cells by integration-defective human immunodeficiency virus type 1 increases de novo methylation. J Virol 2001;75:9753-61.

69 Hayatsu H, Yamashita Y, Yui S, et al. N-Sulfomethylation of guanine, adenine and cytosine with formaldehyde-bisulfite. A selective modification of guanine in DNA. Nucleic Acids Res 1982;10:6281-93.

70 Clark SJ, Harrison J, Paul CL, Frommer M. High sensitivity mapping of methylated cytosines. Nucleic Acids Res 1994;22: 2990-7.

71 Xiong Z, Laird PW. COBRA: a sensitive and quantitative DNA methylation assay. Nucleic Acids Res 1997;25:2532-4.

72 Herman JG, Graff JR, Myohanen S, Nelkin BD, Baylin SB. Methylation-specific PCR: a novel PCR assay for methylation status of CpG islands. Proc Natl Acad Sci U S A 1996;93:98216.

73 Eads CA, Danenberg KD, Kawakami K, et al. MethyLight: a high-throughput assay to measure DNA methylation. Nucleic Acids Res 2000;28:E32.

74 Gonzalgo ML, Jones PA. Quantitative methylation analysis using methylation-sensitive single-nucleotide primer extension (MsSNuPE). Methods 2002;27:128-33.

75 Matin MM, Baumer A, Hornby DP. An analytical method for the detection of methylation differences at specific chromosomal loci using primer extension and ion pair reverse phase HPLC. Hum Mutat 2002;20:305-11.

76 Tost J, Dunker J, Gut IG. Analysis and quantification of multiple methylation variable positions in $\mathrm{CpG}$ islands by Pyrosequencing. Biotechniques 2003;35:152-6.

77 Dupont JM, Tost J, Jammes H, Gut IG. De novo quantitative bisulfite sequencing using the pyrosequencing technology. Anal Biochem 2004;333:119-27.

78 Schatz P, Dietrich D, Schuster M. Rapid analysis of CpG methylation patterns using RNase T1 cleavage and MALDI-TOF. Nucleic Acids Res 2004;32:e167.

79 Ronaghi M, Uhlen M, Nyren P. A sequencing method based on real-time pyrophosphate. Science 1998;281:363, 365.

80 Hayashizaki Y, Hirotsune S, Okazaki Y, et al. Restriction landmark genomic scanning method and its various applications. Electrophoresis 1993;14:251-8.

81 Hayashizaki Y, Hirotsune S, Okazaki Y, et al. A genetic linkage map of the mouse using restriction landmark genomic scanning (RLGS). Genetics 1994;138:1207-38.

82 Okazaki Y, Okuizumi H, Sasaki N, et al. An expanded system of restriction landmark genomic scanning (RLGS Ver. 1.8). Electrophoresis 1995;16:197-202.

83 Kaneda A, Takai D, Kaminishi M, Okochi E, Ushijima T. Methylation-sensitive representational difference analysis and its application to cancer research. Ann N Y Acad Sci 2003;983:13141.

84 Huang TH, Laux DE, Hamlin BC, et al. Identification of DNA methylation markers for human breast carcinomas using the methylation-sensitive restriction fingerprinting technique. Cancer Res 1997;57:1030-4.

85 Yan PS, Chen CM, Shi H, et al. Dissecting complex epigenetic alterations in breast cancer using $\mathrm{CpG}$ island microarrays. Cancer Res 2001;61:8375-80.

86 Yan PS, Wei SH, Huang TH. Differential methylation hybridiza- 
tion using $\mathrm{CpG}$ island arrays. Methods Mol Biol 2002;200:87100.

87 Suzuki Y, Yoshitomo-Nakagawa K, Maruyama K, Suyama A, Sugano S. Construction and characterization of a full lengthenriched and a 5'-end-enriched cDNA library. Gene 1997;200: 149-56.

88 Shi H, Yan PS, Chen CM, Rahmatpanah F, et al. Expressed CpG island sequence tag microarray for dual screening of DNA hypermethylation and gene silencing in cancer cells. Cancer Res 2002;62:3214-20.

89 Weinmann AS, Farnham PJ. Identification of unknown target genes of human transcription factors using chromatin immunoprecipitation. Methods 2002;26:37-47.

90 Leu YW, Yan PS, Fan M, et al. Loss of estrogen signaling leads to epigenetic silencing of its target genes in breast cancer. Cancer Res. 2004;64:8184-92.

91 Cui H, Horon IL, Ohlsson R, Hamilton SR, Feinberg AP. Loss of imprinting in normal tissue of colorectal cancer patients with microsatellite instability. Nat Med 1998;4:1276-80.

92 Verma M, Srivastava S. Epigenetics in cancer: implications for early detection and prevention. Lancet Oncol 2002;3:755-63.

93 Belinsky SA. Gene-promoter hypermethylation as a biomarker in lung cancer. Nat Rev Cancer 2004;4:707-17.

94 Barletta JM, Rainier S, Feinberg AP. Reversal of loss of imprinting in tumor cells by 5-aza-2'-deoxycytidine. Cancer Res 1997; 57:48-50.

95 Claus R, Lubbert M. Epigenetic targets in hematopoietic malignancies. Oncogene 2003;22:6489-96. 